\title{
Cavity-controlled radiative recombination of excitons in thin-film solar cells
}

\author{
Luat T. Vuong, ${ }^{1, a)}$ Gregory Kozyreff, ${ }^{2}$ Rafael Betancur, ${ }^{1}$ and Jordi Martorell ${ }^{1,3, b)}$ \\ ${ }^{1}$ ICFO-Institut de Ciencies Fotoniques, Mediterranean Technology Park, Castelldefels, \\ Barcelona 08860, Spain \\ ${ }^{2}$ Optique Nonlinéaire Théorique, Université Libre de Bruxelles (U.L.B.), Campus de la Plaine, \\ Bruxelles B-1050, Belgium \\ ${ }^{3}$ Departament de Física i Enginyeria Nuclear, Universitat Politècnica de Catalunya, 08222 Terrassa, Spain
}

(Received 8 October 2009; accepted 21 October 2009; published online 7 December 2009)

\begin{abstract}
We study the performance of photovoltaic devices when controlling the exciton radiative recombination time. We demonstrate that when high-quantum-yield fluorescent photovoltaic materials are placed within an optical cavity, the spontaneous emission of the radiative exciton is partially inhibited. The corresponding increase of the exciton lifetime results in an increase of the effective diffusion length and diffusion current. This performance maximizes when the thickness of the cell is comparable to the absorption length. We show that when typical parameter values of thin solar-cell devices are used, the efficiency may improve by as much as three times. (C) 2009 American Institute of Physics. [doi:10.1063/1.3262954]
\end{abstract}

In many photovoltaic devices, a major limitation to high efficiency stems from a short exciton diffusion length given that excitons recombine quickly before reaching the chargeseparation layer. ${ }^{1,2}$ It is assumed possible to reduce some of the recombination rates, except for the radiative one which, in photovoltaics, is generally treated as a material parameter that cannot be changed. However, the radiative rate of recombination is not an immutable property of the radiationmatter interaction, and instead it may be modified by the presence of a cavity or a reflecting surface that alters the electromagnetic environment for the exciton radiation. Indeed, linked to the Purcell effect, ${ }^{3}$ it has been shown that the spontaneous emission rate can be inhibited when a radiative system in the excited state is placed near a reflecting surface ${ }^{4,5}$ in a dielectric slab, ${ }^{6}$ within a Fabry-Pérot type cavity, $^{7,8}$ or photonic crystal. ${ }^{9,10}$ Yet, this very compelling phenomenon has found not many interesting real applications. In photovoltaic materials that exhibit a high-quantumyield fluorescence, where radiative recombination is one of the dominant recombination paths, a reduction of the fluorescence rate may result in a larger exciton diffusion length and ultimately a larger short-circuit current or efficiency.

In the present letter we analyze in detail this effect on the exciton diffusion current when a thin layer of photovoltaic material is placed between two metallic electrodes to form a Schottky diode-type photovoltaic cell. These two parallel electrodes also produce a nonclosed optical cavity for the exciton radiation. One of the electrodes is a perfect conductor with unit reflectivity while the other is a thin metal electrode $^{11}$ with a partial light transmission in order to let the sun photons through but at the same time contribute in forming a cavity for the fluorescence photons. The device configuration that we consider is shown schematically in Fig. 1. The $z$-axis is perpendicular to the plane of the device, the semitransparent metal electrode has thickness $d$ and the active layer has thickness $h$. Inside the active region, excitons are generated and transported via diffusion. Charge separa-

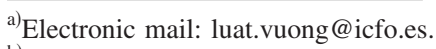

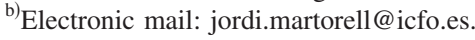

tion occurs at $z=0$ or at the metal electrode with the lower work function.

To determine the diffusion current, we use the FengGhosh model ${ }^{1}$ where we introduce a position-dependent lifetime $\tau(z)$ to account for the cavity-modified exciton recombination rate,

$$
0=D \frac{d^{2} \eta}{d z^{2}}-\frac{\eta}{\tau(z)}+\alpha N \phi|G(z)|^{2},
$$

where $\eta$ is the density of excitons, $D$ is the exciton diffusion coefficient, $\alpha^{-1}$ is the characteristic absorption length, and $\phi$ is the quantum efficiency of the organic dye molecule exciton generation. The photon flux transmitted through the semitransparent electrode is $N$ and the sunlight intensity distribution within the active layer is $|G(z)|^{2}$. We limit the study to photovoltaic materials with high-quantum-yield fluorescence where any mechanism of exciton recombination can be neglected in front of the radiative one.

Prior to solving Eq. (1), we must compute the exciton lifetime $\tau(z)$, which is provided by the position-dependent

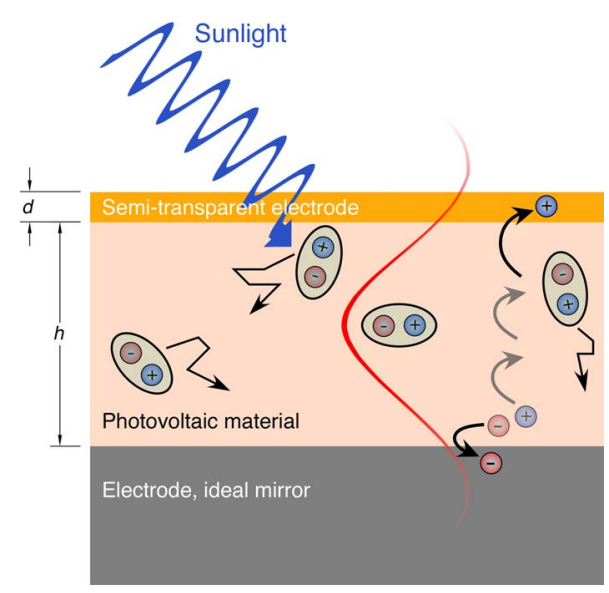

FIG. 1. (Color online) Diagram of the Schottky solar cell. Sunlight produces excitons in the fluorescent active material, which diffuse toward the lower electrode and may recombine via emission of lower energy photons. The red wave indicates pictorially that when the wavelength is larger than the cavity thickness, strong inhibition of radiation is possible. 
spontaneous emission lifetime derived in Ref. 12. The approach employs Fermi's golden rule and assumes that the cavity is a thin and lossy waveguide and that the semitransparent electrode is composed of a conducting material. This reference studies the spontaneous emission rates in an asymmetric nonideal cavity, which is the prototypical architecture of a solar-cell device. The radiative decay rates associated with dipoles aligned parallel and perpendicular to the electrodes $\Gamma_{\|}$and $\Gamma_{\perp}$ are

$$
\begin{aligned}
\frac{\Gamma_{\|}}{\Gamma_{0}}= & \frac{3}{2 k}\left[\int_{0}^{k} \frac{\left|k_{z} \sin \left(q_{1} z\right)\right|^{2} d k_{z}}{\left|q_{2} \rho_{E} \sin \left(q_{1} h-\beta_{E}\right)\right|^{2}}\right. \\
& \left.+\frac{1}{k^{2}} \int_{0}^{k} \frac{\left|q_{2} \sin \left(q_{1} z\right)\right|^{2} d k_{z}}{\left|\tilde{n}_{2}^{2} \rho_{M} \sin \left(q_{1} h+\beta_{M}\right)\right|^{2}}\right], \\
\frac{\Gamma_{\perp}}{\Gamma_{0}}= & \frac{3}{k^{3}} \int_{0}^{k} \frac{\left(k^{2}-k_{z}^{2}\right)\left|Q_{M} \cos \left(q_{1} z\right)\right|^{2} d k_{z}}{\left|\tilde{n}_{1}^{2} \rho_{M} \sin \left(q_{1} h+\beta_{M}\right)\right|^{2}},
\end{aligned}
$$

where $k=2 \pi / \lambda$ is the wavenumber, $\Gamma_{0}$ is the decay rate in a homogeneous bulk material, $q_{m}^{2}=\left(\widetilde{n}_{m}^{2}-1\right) k^{2}+k_{z}^{2}$ are the $z$-component wavenumbers and $\widetilde{n}_{m}$ are the complex refractive indices for the active layer $(m=1)$ and semitransparent electrode $(m=2)$. In addition,

$$
\left.\begin{array}{l}
\tan \left(\beta_{Z}\right)=Q_{Z} \cot \left(q_{2} d\right), \\
\rho_{Z}=\sin ^{2}\left(q_{2} d\right)+Q_{Z}^{2} \cos ^{2}\left(q_{2} d\right),
\end{array}\right\} Z=E, M,
$$

where $Q_{E}=q_{1} / q_{2}$ and $Q_{M}=\widetilde{n}_{1}^{2} q_{2} / \tilde{n}_{2}^{2} q_{1}$.

Associated with the spontaneous emission rates $\Gamma_{\perp}$ and $\Gamma_{\|}$are the radiative lifetimes $\tau_{\perp}(z)$ and $\tau_{\|}(z)$. A randomly oriented dipole has an average lifetime $\tau_{\text {avg }}(z)=\left(2 / 3 \Gamma_{\|}\right.$ $\left.+1 / 3 \Gamma_{\perp}\right)^{-1}$. In a numerical application of Eqs. (2) and (3), we determine the corresponding radiative lifetimes under the assumption that the active material refractive index, absorption, and quantum yield are $\widetilde{n}_{1}=1.6+0.8 i, \alpha^{-1}=50 \mathrm{~nm}$, and $\phi=1$ with an emission wavelength $\lambda=600 \mathrm{~nm}$. We use a diffusion coefficient of $D=5 \times 10^{-9} \mathrm{~m}^{2} / \mathrm{s}$ and a free-space diffusion length of $L_{\text {diff }}=\sqrt{D / \Gamma_{0}}=5 \mathrm{~nm}$. These provide approximate values for a range of organic photovoltaic materials. In addition, we assume that the semitransparent electrode has a complex refractive index of $\widetilde{n}_{2}=1+3 i$. The imaginary part of this refractive index corresponds to some higherwork-function metals that could be used as the semitransparent electrode. ${ }^{13}$ As seen in Fig. 2, for this nonsymmetric cavity we observe that in thinner devices $h<30 \mathrm{~nm}$, inhibition is stronger for dipoles aligned parallel compared to dipoles aligned plane-perpendicular. The lifetime for a planeparallel dipole varies orders of magnitude and increases sharply near the electrode at $z=0$. For thicker devices $h>30 \mathrm{~nm}$, the lifetimes associated with plane-perpendicular dipoles are longer at the semitransparent electrode. Note, however, that the spontaneous emission is inhibited at all positions for either dipole polarization.

To solve Eq. (1) we assume that the electrode behaves as a perfect surface sink and subsequently, the exciton density at the electrodes is zero or $\eta(z=0)=\eta(z=h)=0$. The exciton diffusion current, neglecting the electrode resistance, is $J_{\text {diff }}=D d \eta / d z$ and is associated with the short-circuit current when the active material is also a good hole conductor. Here, we initially distill the cavity-induced inhibition effect on the exciton diffusion by neglecting the multiple-reflections of solar photons and sunlight absorption of the thin metal elec-

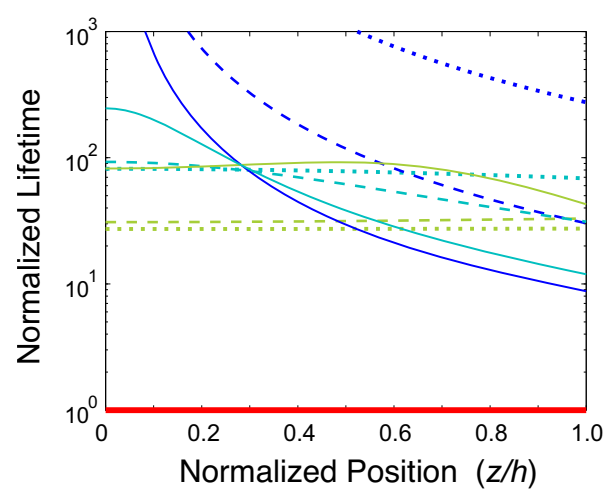

FIG. 2. (Color online) (a) Normalized radiative lifetime as a function of position for different active-layer thicknesses $h=100$ (solid), 30 (dashed), and $10 \mathrm{~nm}$ (dotted). Blue curves: $\Gamma_{0} / \Gamma_{\|}(z)$, green curves: $\Gamma_{0} / \Gamma_{\perp}(z)$, cyan curves: $\Gamma_{0} /\left[2 / 3 \Gamma_{\|}(z)+1 / 3 \Gamma_{\perp}(z)\right]$. The red line denotes the bulk-material rate. The electrode thickness is $d=5 \mathrm{~nm}$.

trode. Only afterwards do we incorporate these effects into our analysis and compare the cavity-controlled photovoltaic cell with a cell in which a nonfluorescent material and a highly transparent conductive oxide is used.

Accordingly in our initial numerical application of the solution to Eq. (1) we employ the simple intensity distribution $|G(z)|^{2}=\exp [-\alpha(h-z)]$ and fix the photon flux $N$ to correspond to a sunlight intensity of $1 \mathrm{~kW} / \mathrm{m}^{2}$. In Fig. 3 the calculated exciton diffusion currents are shown as a function of device active-layer thicknesses. The red line shows the performance of a solar device in which the active material has a constant lifetime given by its bulk properties i.e., a nonfluorescent active material with the same dipole strength, whose exciton lifetime is constant $\tau(z)=1 / \Gamma_{0}$. For the fluorescent dipole we observe that when we increase the thickness of the semitransparent electrode, the thin solar device behaves increasingly as an optical cavity and device performance improves. An increase of the exciton lifetime due to inhibited spontaneous emission coincides with a broadening and shift of the peak in the $J_{\text {diff }}$ versus $h$ curve. Note that maximum diffusion current is achieved when the active-layer thickness becomes comparable to the absorption length. When the semitransparent electrode is 20 -nm-thick, the peak current density increases fivefold. As much as a factor of 8 improvement is observed if one controls the orientation of dipoles in the active layer.

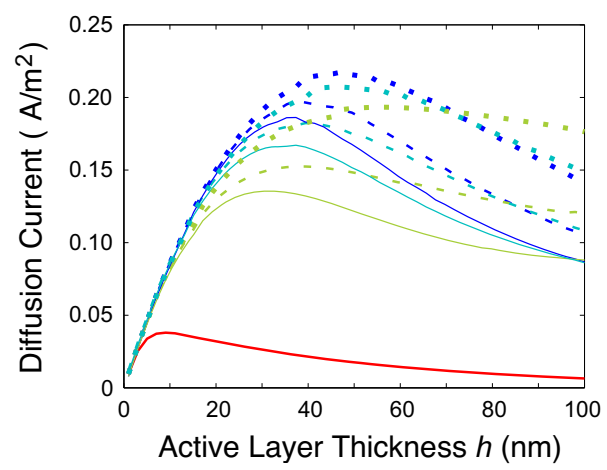

FIG. 3. (Color online) Calculated solar-cell exciton diffusion current for active-layer thicknesses $h<100 \mathrm{~nm}$. Blue curves: $\tau(z)=1 / \Gamma_{\|}(z)$, green curves: $\tau(z)=1 / \Gamma_{\perp}(z)$, cyan curves: $\tau(z)=1 /\left(2 / 3 \Gamma_{\|}(z)+1 / 3 \Gamma_{\perp}(z)\right)$, and red curve: $1 / \Gamma_{0}$. The electrode thickness varies $d=5 \mathrm{~nm}$ (solid), $d=10 \mathrm{~nm}$ (dashed), and $d=20 \mathrm{~nm}$ (dotted). 


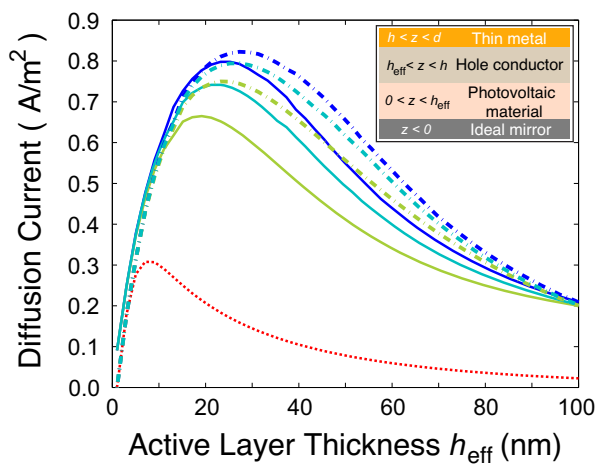

FIG. 4. (Color online) Inset: diagram of a modified Schottky solar cell where a hole conductor of variable thickness $h-h_{\text {eff }}$ is inserted between the semitransparent conducting electrode and active layer with thickness $h_{\text {eff }}$. Calculated solar-cell exciton diffusion current for a $74 \%$-transmissive electrode of thickness $d=5 \mathrm{~nm}$ and active-layer thicknesses $h, h_{\text {eff }}<100 \mathrm{~nm}$ without (solid line) and with the hole conductor (dash-dotted line). Blue curves: $\tau(z)=1 / \Gamma_{\|}(z)$, green curves: $\tau(z)=1 / \Gamma_{\perp}(z)$, cyan curves: $\tau(z)$ $=1 /\left[2 / 3 \Gamma_{\|}(z)+1 / 3 \Gamma_{\perp}(z)\right]$. The dotted red curve assumes an electrode with $80 \%$ transmission and a constant rate $1 / \Gamma_{0}$.

Typically, such enhanced performance due to cavity effects cannot occur without also reducing the sunlight that is transmitted through the electrode. Additionally, sunlight multiple reflections may modify the enhancement seen at the fluorescence wavelength. In our last numerical application of the solution to Eq. (1) we employ the intensity distribution

$$
|G(z)|^{2}=\left|\frac{e^{-\alpha(h-z) / 2}+e^{-\alpha(h+z) / 2}}{1-\operatorname{Re}^{-h \alpha}}\right|^{2},
$$

which assumes incoherent light and a sunlight reflectivity at the active-layer electrode interface $R=\left(\widetilde{n}_{2}-\widetilde{n}_{1}\right) /\left(\widetilde{n}_{2}+\widetilde{n}_{1}\right)$. We also consider the finite absorption of the semitransparent electrode of thickness $d=5 \mathrm{~nm} .{ }^{11}$ The inclusion of sunlight interference effects ${ }^{14,15}$ are neglected because they do not change the illustrated trends.

In Fig. 4, we show the corresponding exciton diffusion current as a function of active layer thickness and solid lines illustrate the variable recombination rate in a single-layer solar device. The dotted red lines illustrate a constant recombination rate $\Gamma_{0}$ with a reduced flux of $80 \%$ associated with a highly transparent electrode such as indium tin oxide. The dotted red line accounts for reflections and a comparison between the peak of the solid cyan line to that of the dotted red line indicates that as much as a factor of 2.7 enhancement in the average solar cell performance can be expected.

It is possible to achieve a $10 \%$ improvement over this factor by adding a nonfluorescent hole conductor that takes advantage of the fact that the larger distance between electrodes extends the radiative lifetimes [cf. Fig. 2]. In this modified geometry, the active-layer thickness is varied but the distance between the electrodes $h$ is fixed at $100 \mathrm{~nm}$, where a nonabsorbing hole-conducting layer is added between the active material and electrodes $h_{\mathrm{eff}}<z<h$, as illustrated in the inset illustration of Fig. 4. If a smaller diffusion coefficient $D$ or larger absorption length $\alpha^{-1}$ is employed, the advantage of adding a hole conductor becomes more apparent. Moreover, under these conditions, the effect of dipole orientation plays a larger role. This dependence highlights the importance of understanding the active material morphology for modeling exciton transport.

To conclude, the use of photovoltaic materials that may exhibit a large quantum-yield fluorescence may significantly enhance the performance of excitonic photovoltaic cells where the exciton diffusion length is shorter than the absorption length. The enhancement we observe is obtained when the photovoltaic material is placed within a nonideal and nonsymmetric planar optical cavity. We demonstrate that the effect of such cavity is to inhibit the spontaneous emission of the radiative excitons. When a thin metal electrode is used as the semitransparent end of such cavity, the increase in exciton diffusion current is close to threefold. However, this is not the absolute limit, and further improvements may be expected if other cavity designs are considered, such as, for instance the use of one- or two-dimensional photonic crystals that could be made highly transparent to the absorption band but reflecting to the fluorescence emission band of the photovoltaic material.

This work has been supported by the Spanish Ministerio de Ciencia e Inovacin, under Grants MAT2008-00910/NAN and CONSOLIDER NANOLIGHT Grant No. CSD200700046. L.T.V. is supported by a Marie Curie grant (Grant No. PIIF-GA-2009-236566) administered by the European Commission. G.K. is a Research Associate of the F.R.S-FNRS (Belgium).

${ }^{1}$ A. K. Ghosh and T. Feng, J. Appl. Phys. 49, 5982 (1978).

${ }^{2}$ P. Peumans, A. Yakimov, and S. R. Forrest, J. Appl. Phys. 93, 3693 (2003)

${ }^{3}$ E. M. Purcell, Phys. Rev. 69, 37 (1946).

${ }^{4}$ H. Morawitz, Phys. Rev. 187, 1792 (1969).

${ }^{5}$ K. H. Drexhage, Prog. Opt. 12, 165 (1974).

${ }^{6}$ H. P. Urbach and G. L. J. A. Rikken, Phys. Rev. A 57, 3913 (1998).

${ }^{7}$ R. G. Hulet, E. S. Hilfer, and D. Kleppner, Phys. Rev. Lett. 55, 2137 (1985).

${ }^{8}$ S. D. Brorson, H. Yokoyama, and E. P. Ippen, IEEE J. Quantum Electron. 26, 1492 (1990).

${ }^{9}$ E. Yablonovitch, Phys. Rev. Lett. 58, 2059 (1987).

${ }^{10}$ J. Martorell and M. Lawandy, Phys. Rev. Lett. 65, 1877 (1990).

${ }^{11}$ D. S. Ghosh, L. Martnez, S. Giurgulo, P. Vergani, and V. Pruneri, Opt Lett. 34, 325 (2009).

${ }^{12} \mathrm{G}$. Kozyreff (unpublished).

${ }^{13}$ P. B. Johnson and R. W. Christy, Phys. Rev. B 6, 4370 (1972).

${ }^{14}$ S. Rim and P. Peumans, J. Appl. Phys. 103, 124515 (2008).

${ }^{15}$ L. Yang, H. Chen, and M. Wang, Thin Solid Films 516, 7701 (2008). 